\title{
An Upper Bound on the Radius of a 3-Vertex-Connected $\mathrm{C}_{4}$-Free Graph
}

\author{
Blessings T. Fundikwa $\mathbb{D}^{1},{ }^{1}$ Jaya P. Mazorodze, ${ }^{1}$ and Simon Mukwembi ${ }^{2}$ \\ ${ }^{1}$ Department of Mathematics, University of Zimbabwe, Harare, Zimbabwe \\ ${ }^{2}$ School of Mathematics, Statistics and Computer Science, University of KwaZulu-Natal, Durban, South Africa
}

Correspondence should be addressed to Blessings T. Fundikwa; takudzwabf@gmail.com

Received 30 May 2020; Accepted 15 July 2020; Published 4 August 2020

Academic Editor: Elena Guardo

Copyright $\odot 2020$ Blessings T. Fundikwa et al. This is an open access article distributed under the Creative Commons Attribution License, which permits unrestricted use, distribution, and reproduction in any medium, provided the original work is properly cited.

We show that if $G$ is a 3 -vertex-connected $C_{4}$-free graph of order $n$ and radius $r$, then the inequality $r \leq(2 n / 9)+O(1)$ holds. Moreover, graphs are constructed to show that the bounds are asymptotically sharp.

\section{Introduction}

Let $G=(V, E)$ be a finite, connected, undirected graph with vertex set $V$ and edge set $E$. The distance $d_{G}(u, v)$ between two vertices $u, v$ of $G$ is the length of a shortest $u-v$ path in $G$. The eccentricity ec $(v)$ of a vertex $v \in V$ is the maximum distance between $v$ and any other vertex in $G$. The value of the minimum eccentricity of the vertices of $G$ is called the radius of $G$ denoted by $\operatorname{rad}(G)$. The degree $\operatorname{deg}(v)$ of a vertex $v$ of $G$ is the number of edges incident with $v$. The minimum degree $\delta(G)$ is the minimum of the degrees of vertices in $G$. The open neighbourhood $N(v)$ of a vertex $v$ is the set of all vertices of $G$ adjacent to $v$. The closed neighbourhood $N[v]$ of $v$ is the set $N(v) \cup\{v\}$. A graph is triangle-free if it does not contain $C_{3}$ as a subgraph and $C_{4}$ - free if it does not contain $\mathrm{C}_{4}$ as a subgraph. For notions not defined, here we refer the reader to [1].

In our research, we are concerned with upper bounds on radius in terms of order and each of the three classical connectivity measures, namely, minimum degree, edgeconnectivity, and vertex-connectivity for general graphs and for graphs with forbidden subgraphs such as $C_{3}$ and $C_{4}$. Several upper bounds on radius in terms of other graph parameters are known. For example, Erdös et al. [2] and independently Dankelmann et al. [3] proved that if $G$ is a connected graph of order $n$ and minimum degree $\delta \geq 2$, then

$$
\operatorname{rad}(G) \leq \frac{3 n}{2(\delta+1)}+O(1) .
$$

Erdös et al. [2] proved that if $G$ is a connected trianglefree graph of order $n$ and minimum degree $\delta \geq 2$, then

$$
\operatorname{rad}(G) \leq \frac{n-2}{\delta}+12,
$$

and that if $G$ is a connected $C_{4}$-free graph of order $n$ and minimum degree $\delta \geq 2$, then

$$
\operatorname{rad}(G) \leq \frac{5 n}{2\left(\delta^{2}-2[\delta / 2]+1\right)}
$$

Bounds on the radius in terms of order and edge-connectivity were given by Dankelmann et al. [4]. They showed that if $\lambda=3$, then

$$
\operatorname{rad}(G) \leq \frac{n}{3}+\frac{17}{3},
$$

and they constructed graphs that, apart from the additive constant, attain the bound. Mukwembi, in [5], showed that if $G$ is a triangle-free 3-edge-connected graph, then

$$
\operatorname{rad}(G) \leq \frac{3 n}{10}+\frac{56}{5},
$$

and constructed graphs that, apart from the additive constant, attain the bound. Bounds on the radius in terms of 
order and vertex-connectivity were given by Harant and Walther [6]. If $\kappa(G)$ is even, then the well-known bound, $\operatorname{diam}(G) \leq(n+\kappa(G)-2) / \kappa(G)$, on the diameter is also sharp for the radius. For odd $\kappa(G)$, Harant and Walther [6] showed that

$$
\operatorname{rad}(G) \leq \frac{n}{\kappa(G)+1}+O(\log n) .
$$

In [7], Harant showed that for $\kappa=3$, the $O(\log n)$ term can be replaced by a constant. It is worth noting that, while it has not been formally communicated, the bounds on the radius of triangle-free graphs with respect to order and vertex-connectivity are exactly the same as those for graphs without restrictions where subgraphs are concerned. To date, there are no known bounds reported on the radius of $\mathrm{C}_{4}$-free graphs with respect to order and vertex-connectivity. The main theorem of this paper seeks to prove the following:

Theorem 1. Let $G$ be a 3-vertex-connected $C_{4}$-free graph of $\operatorname{order} n$. Then, $\operatorname{rad}(G) \leq(2 n / 9)+(263 / 9)$.

To show that this bound is asymptotically sharp, consider the graph $C_{k S}$ where $S$ is the graph in Figure 1 and $C_{k S}$ is the graph formed by replacing each vertex of a $C_{k}$-cycle by the graph $S$ and replacing an edge between consecutive vertices in the cycle by an edge connecting $y$ to $v$ and another edge connecting $x$ to $u$, and this is between the two copies of $S$ that have replaced consecutive vertices in $C_{k}$. As an example, consider $C_{4 S}$ in Figure 2. Note that $C_{k S}$ is a 3-vertex-connected $C_{4}$-free graph and that whenever $k \equiv 0 \bmod 2$, we have

$$
\operatorname{rad}\left(C_{k S}\right)=\frac{2 n}{9} .
$$

\section{Results}

Let $G$ be a 3-vertex-connected, $C_{4}$-free graph, of order $n$, and $z$ be a fixed centre vertex of $G$, so that $\operatorname{rad}(G)=r=\mathrm{ec}(z)$. For each $i=0,1,2, \ldots, r$, let $N_{i}:=\left\{v \in V(G) \mid d_{G}(v, z)=i\right\}$ and $k_{i}=\left|N_{i}\right|$. Note that for each $i$ with $1 \leq i \leq r-1$ and any $v \in N_{i}$, we have $N(v) \subseteq N_{i-1} \cup N_{i} \cup N_{i+1}$. We employ the notation $N_{\leq j}=\cup_{0 \leq i \leq j} N_{i}$ and $N_{\geq j}=\cup_{j \leq i \leq r} N_{i}$. Since $N_{r} \neq \varnothing$, from now onwards fix a vertex $z_{r} \in N_{r}$. Form a spanning tree $T$ of $G$ that is distance preserving from $z$. For a vertex $y \in V(G)$, denote by $T(z, y)$, the path connecting $z$ and $y$ in $T$. We say $y$ is related to $z_{r}$ if there exist vertices $u, v \in V(G) \quad$ where $u \in V\left(T\left(z, z_{r}\right)\right) \cap N_{>37} \quad$ and $v \in V(T(z, y)) \cap N_{\geq 37}$ such that $d_{G}(u, v) \leq 18$.

Lemma 1. Let $r \geq 73$ and $z, z_{r}$ as above. Then, there exists a vertex in $N_{\geq r-37}$ which is not related to $z_{r}$.

Proof. Suppose to the contrary that every vertex $y \in N_{\geq r-37}$ is related to $z_{r}$. Let $z_{37}$ be the vertex of $T\left(z, z_{r}\right)$ which belongs to $N_{37}$. Then, for any $x \in N_{\leq r-38}$, we have $d_{G}\left(z_{37}, x\right) \leq d_{G}\left(z_{37}, z\right)+d_{G}(z, x) \leq 37+r-38=r-1$.

Since every vertex in $N_{\geq r-37}$ is related to $z_{r}$, for any $x \in N_{\geq r-37}$, there exist $u$, $v$, where $u \in V\left(T\left(z, z_{r}\right)\right) \cap N_{\geq 37}$ and $v \in V(T(z, x)) \cap N_{\geq 37}$ such that $d_{G}(u, v) \leq 18$. Thus,

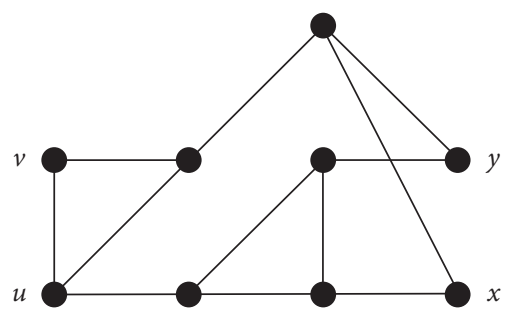

FIGURE 1: The graph S.

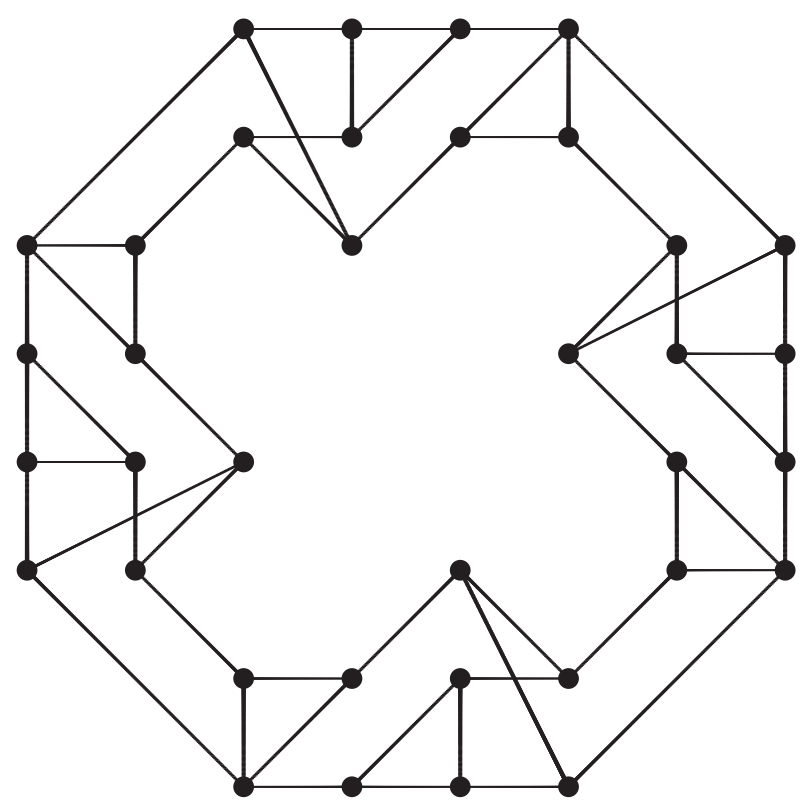

Figure 2: $C_{4 S}$.

$d_{G}\left(z_{37}, x\right) \leq d_{G}\left(z_{37}, u\right)+d_{G}(u, v)+d_{G}(v, x) \leq d_{G}(z, u)-37+$ $18+\left(r-d_{G}(z, v)\right) \leq r-19+d_{G}(u, v) \leq r-1$, for any $x \in$ $N_{\geq r-37}$. Hence, ec $\left(z_{19}\right) \leq r-1$, contradicting the fact that $\operatorname{rad}(G)=r$.

Throughout the rest of this paper, $y_{t}$ is a fixed vertex in $N_{\geq r-37}$ which is not related to $z_{r}$. Let $T\left(z, z_{r}\right)=z x_{1} x_{2} \ldots$ $x_{r-1} z_{r}$ (respectively, $\left.T\left(z, y_{t}\right)=z y_{1} y_{2} \ldots y_{t-1} y_{t}\right)$ be a path from $z$ to $z_{r}$ (respectively, $y_{t}$ ) in $T$. For each $i$ with $0 \leq i \leq r$ $(0 \leq i \leq r-37)$ denote by $X_{i}^{(d)}\left(Y_{i}^{(d)}\right), d \in\{0,1,2,3,4,5$, $6,7,8,9\}$, the set of all vertices in $N_{i}$ whose distance from $V\left(T\left(z, z_{r}\right)\right) \cap N_{\geq 37}\left(V\left(T\left(z, y_{t}\right) \cap N_{\geq 37}\right)\right)$ is at most $d$ in $G$. Thus, for example, $X_{i}^{(3)} \subseteq X_{i}^{(4)}$. For each $i$ with $0 \leq i \leq r$ $(0 \leq i \leq r-37)$ denoted by $X_{i}^{[e]}\left(Y_{i}^{[e]}\right), e \in\{1,2,3,4,5,6,7$, $8,9\}$, the set of all vertices in $N_{i}$ whose distance from $V\left(T\left(z, z_{r}\right)\right) \cap N_{\geq 37}\left(V\left(T\left(z, y_{t}\right) \cap N_{\geq 37}\right)\right)$ is exactly $e$ in $G$. Thus, for example, $X_{i}^{(3)}=X_{i}^{[0]} \uplus X_{i}^{[1]} \uplus X_{i}^{[2]} \uplus X_{i}^{[3]}$. For each $i$ with $0 \leq i \leq r(0 \leq i \leq r-37)$ denoted by $X_{i}^{[d, e]}\left(Y_{i}^{[d, e]}\right)$, $d, e \in\{1,2,3,4,5,6,7,8,9\}$, the set of all vertices in $N_{i}$ whose distance from $V\left(T\left(z, z_{r}\right)\right) \cap N_{\geq 37}\left(V\left(T\left(z, y_{t}\right) \cap N_{\geq 37}\right)\right)$ is $f$ where $d \leq f \leq e$ in $G$. Thus, for example, $X_{i}^{[1,3]}=X_{i}^{[1]} \uplus$ $X_{i}^{[2]} \uplus X_{i}^{[3]}$. For each $d \in\{2,3\}$ denoted by $Q_{\leq d}[a]$, the set of all vertices in $G$ whose distance from $a \in V(G)$ is at most $d$ in $G$. Thus, for example, $Q_{\leq 2}[a] \subseteq Q_{\leq 3}[a]$. The following fact follows from the fact that $y_{t}$ is not related to $z_{r}$ : 
Fact 1. $\left(\cup_{i=37}^{r-37} X_{i}^{(9)}\right) \cap\left(\cup_{i=37}^{r-37} Y_{i}^{(9)}\right)=\varnothing$.

Lemma 2. Let $r \geq 81$. With the above notation, for each $i \in\{37,38, \ldots, r-40\},\left|\cup_{j=i}^{i+3} X_{j}^{(2)}\right| \geq 6$ (respectively, $\mid \cup_{j=i}^{i+3} Y_{j}^{(2)}$ $\mid \geq 6$ ).

Proof. We show that the result holds for $\left|\cup_{j=i}^{i+3} X_{j}^{(2)}\right|$ as it follows analogously on $\left|\cup_{j=i}^{i+3} Y_{j}^{(2)}\right|$. Clearly, $x_{i+1} \in X_{i+1}^{(0)} \subseteq X_{i+1}^{(2)}$, $x_{i+2} \in X_{i+2}^{(0)} \subseteq X_{i+2}^{(2)}$ and $x_{i+1} x_{i+2} \in E(G)$. Also, $\operatorname{deg}\left(x_{i+1}\right)$, $\operatorname{deg}\left(x_{i+2}\right) \geq \delta(G) \geq \kappa(G)=3$. Furthermore, $N\left(x_{i+1}\right) \cup N$ $\left(x_{i+2}\right) \subseteq \cup_{j=i}^{i+3} X_{j}^{(1)}$, and each pair of vertices (in this case $x_{i+1}$ and $x_{i+2}$ ) shares at most one neighbour since $G$ is $C_{4}$-free. There are two cases to consider:

Case 1: suppose that $x_{i+1}$ and $x_{i+2}$ do not share a neighbour. Then, $\left|\cup_{j=i}^{i+3} X_{j}^{(2)}\right| \geq\left|\cup{ }_{j=i}^{i+3} X_{j}^{(1)}\right| \geq \mid N\left(x_{i+1}\right)$ $\uplus N\left(x_{i+2}\right) \mid \geq 3+3=6$, and we are done.

Case 2: assume that $x_{i+1}$ and $x_{i+2}$ share a neighbour, say $a$. Note that $a \notin X_{i}^{(2)} \cup X_{i+3}^{(2)}$ since $\left(X_{i}^{(2)} \cap N\left[x_{i+2}\right]\right) \cup$ $\left(X_{i+3}^{(2)} \cap N\left[x_{i+1}\right]\right)=\varnothing$. From this, it becomes obvious that $a \notin\left\{x_{i}, x_{i+3}\right\}$. Thus, since $a$ is adjacent to $x_{i+1}$ and $x_{i+2}$, we realise that $a \in X_{i+1}^{(1)} \cup X_{i+2}^{(1)}$. Now, $\left\{a, x_{i}, x_{i+1}, x_{i+2}, x_{i+3}\right\} \subseteq \cup_{j=i}^{i+3} X_{j}^{(1)} \subseteq \cup_{j=i}^{i+3} X_{j}^{(2)}$. Observe that since $\operatorname{deg}(a) \geq \delta(G) \geq \kappa(G)=3$, $a$ has at least one neighbour $b \notin\left\{x_{i+1}, x_{i+2}\right\}$. Again since $\left\{a x_{i+1}, a x_{i+2}\right.$, $\left.x_{i} x_{i+1}, x_{i+1} x_{i+2}, x_{i+2} x_{i+3}\right\} \subseteq E(G)$ and because $G$ is $C_{4}$-free, we see that $b \notin\left\{x_{i}, x_{i+3}\right\}$. Because $a b \in E(G)$ and $a \in X_{i+1}^{(1)} \cup X_{i+2}^{(1)}$, we see that $b \in \cup_{j=i}^{i+3} X_{j}^{(2)}$. Therefore, $\left|\cup_{j=i}^{i+3} X_{j}^{(2)}\right| \geq\left|\{b\} \uplus\left\{a, x_{i}, x_{i+1}, x_{i+2}, x_{i+3}\right\}\right|=6$, which settles Lemma 2.

For $i \in\{37,38, \ldots, r-40\}$, let $S$ be an induced subgraph of $G$ such that $V(S)=\cup_{j=i}^{i+3} X_{j}^{(9)}$ (respectively, $V(S)=$ $\left.\cup_{j=i}^{i+3} Y_{j}^{(9)}\right)$ and $H$ be an induced subgraph of $S$. Then, we have the following definitions.

Definition 1. $S$ is a scant subgraph of $G$ (or simply, $S$ is scant) if $|V(S)| \leq 8$.

Definition 2. We say $H$ is an attached subgraph of $S$ (or simply, $H$ is attached) if there exists a path in $H$ connecting at least one vertex $u \in X_{i}^{(9)}$ (respectively, $u \in Y_{i}^{(9)}$ ) to at least one vertex $v \in X_{i+3}^{(9)}$ (respectively, $v \in Y_{i+3}^{(9)}$ ), otherwise $H$ is a detached subgraph of $S$ (or simply, $H$ is detached).

Definition 3. $S$ is said to be a detachable subgraph of $G$ (or simply, $S$ is detachable) if there exists a vertex $v \in V(S)$ such that $H=S-v$ is a detached subgraph of $S$.

\section{Lemma 3. If $S$ is scant, then it is detachable.}

Proof. We show that the result holds for $S=\cup_{j=i}^{i+3} X_{j}^{(9)}$ as it follows analogously on $S=\cup_{j=i}^{i+3} Y_{j}^{(9)}$. Note that if $H=S-X_{j}^{(9)}, j \in\{i, i+1, i+2, i+3\}$, then $H$ is detached. Thus, if $1 \in\left\{\left|X_{j}^{(9)}\right|: j \in\{i, i+1, i+2, i+3\}\right\}$, we immediately conclude that $S$ is detachable. So, suppose that $\left|X_{j}^{(9)}\right| \geq 2$ whenever $j \in\{i, i+1, i+2, i+3\}$. Now, because of our supposition, if $\left|X_{j}^{(9)}\right| \geq 3$ for any $j \in\{i, i+1, i+2, i+3\}$, then $\left|\cup_{j=i}^{i+3} X_{j}^{(9)}\right| \geq 9$ contradicting the condition of this lemma. Thus, $\left|X_{j}^{(9)}\right|<3$ whenever $j \in\{i, i+1, i+2, i+3\}$, and consequently, $\left|X_{i}^{(9)}\right|=\left|X_{i+1}^{(9)}\right|=\left|X_{i+2}^{(9)}\right|=\left|X_{i+3}^{(9)}\right|=2$. Accordingly, $\left|X_{i}^{(9)}\right|+\left|X_{i+1}^{(9)}\right|+\left|X_{i+2}^{(9)}\right|=6$ and $\left|X_{i+1}^{(9)}\right|+\left|X_{i+2}^{(9)}\right|+\left|X_{i+3}^{(9)}\right|=6$. For $j \in\{i, i+1, i+2, i+3\}$, let $a_{j}$ be the other vertex in $X_{j}^{(9)}$ which is not $x_{j}$. Note that a path $P=a_{i} a_{i+1} a_{i+2} a_{i+3}$ such that $\left\{a_{i}, a_{i+1}, a_{i+2}, a_{i+3}\right\} \subseteq \cup_{j=i}^{i+3} X_{j}^{[8,9]}$ does not exist since $G-\left\{x_{i}, x_{i+3}\right\}$ would be disconnected, thereby contradicting $\kappa=3$. If $a_{i+1} \in X_{i+1}^{[9]}$ or $a_{i+2} \in X_{i+2}^{[9]}$, then $S$ is detachable since $H=S-x_{i+1}$ or $H=S-x_{i+2}$ is detached. So, suppose $a_{i+1} \in X_{i+1}^{(8)}$ and $a_{i+2} \in X_{i+2}^{(8)}$. If $a_{i+1} x_{i+1} \notin E(G)$, then since $a_{i+1}$ and $x_{i+1}$ can share at most one neighbour and $\delta \geq 3$, $\left|\cup{ }_{j=i}^{i+2} X_{j}^{(9)}\right| \geq\left|N\left[a_{i+1}\right] \cup N\left[x_{i+1}\right]\right| \geq 7$ immediately contradicting $\left|X_{i}^{(9)}\right|+\left|X_{i+1}^{(9)}\right|+\left|X_{i+2}^{(9)}\right|=6$. Thus, $a_{i+1} x_{i+1} \in E(G)$. From a similar argument, we obtain $a_{i+2} x_{i+2} \in E(G)$. Note that $a_{i+2}$ is necessarily adjacent to at least one vertex in $X_{i+1}^{(9)}$. Observe that $a_{i+1} a_{i+2} \notin E(G)$, otherwise $\left\{a_{i+1} a_{i+2}, a_{i+2} x_{i+2}\right.$, $\left.x_{i+2} x_{i+1}, x_{i+1} a_{i+1}\right\}$ would form a $C_{4}$-subgraph, which is illegal. Thus, $a_{i+2}$ can only be adjacent to $x_{i+1} \in X_{i+1}^{(9)}$. Consider $x_{i+2}$. Note that $a_{i+1} x_{i+2} \notin E(G)$, otherwise $\left\{a_{i+1} x_{i+2}, x_{i+2} a_{i+2}\right.$, $\left.a_{i+2} x_{i+1}, x_{i+1} a_{i+1}\right\}$ would form a $C_{4}$-subgraph, which is forbidden. From the above, we see that $H=S-x_{i+1}$ is detached, and thus, $S$ is detachable and Lemma 3 is settled.

Lemma 4. If $S$ is scant, then $\cup_{j=i+1}^{i+3} X_{j}^{[6]}=\varnothing$ (respectively, $\left.\cup_{j=i+1}^{i+3} Y_{j}^{[6]}=\varnothing\right)$.

Proof. We show that the result holds for $S=\cup_{j=i}^{i+3} X_{j}^{(9)}$ as it follows analogously on $S=\cup_{j=i}^{i+3} Y_{j}^{(9)}$. Suppose to the contrary that there exists $a \in \cup_{j=i+1}^{i+3} X_{j}^{[6]}$. There are two cases to consider:

(i) Case 1: suppose $a \in X_{i+1}^{[6]} \cup X_{i+2}^{[6]}$. This means $a \in X_{i+1}^{[6]}$ or $X_{i+2}^{[6]}$. If $a \in X_{i+1}^{[6]}\left(a \in X_{i+2}^{[6]}\right)$, then $N[a] \cap N\left[x_{i+1}\right]=$ $\varnothing \quad\left(N[a] \cap N\left[x_{i+2}\right]=\varnothing\right)$ yielding $\left|\cup_{j=i}^{i+2} X_{j}^{(9)}\right| \geq$ $\left|N[a] \uplus N\left[x_{i+1}\right]\right| \geq 8\left(\left|\cup_{j=i+1}^{i+3} X_{j}^{(9)}\right| \geq\left|N[a] \uplus N\left[x_{i+2}\right]\right|\right.$ $\geq 8)$. Thus, since $\left|X_{i+3}^{(9)}\right| \geq 1 \quad\left(\left|X_{i}^{(9)}\right| \geq 1\right)$, we obtain $\left|\cup_{j=i}^{i+3} X_{j}^{(9)}\right| \geq 8+1=9 \quad\left(\left|\cup_{j=i}^{i+3} X_{j}^{(9)}\right| \geq 1+8=9\right)$ contradicting our assertion that $S$ is scant.

Case 2: suppose $a \in X_{i+3}^{[6]}$. If this is so, then $a$ is necessarily adjacent to some $b \in N_{i+2}$. More specifically, $b \in X_{i+2}^{[5,7]}$. Observe that $N[b] \cap N\left[x_{i+2}\right]=\varnothing$. Thus, $\left|\cup \underset{j=i+1}{i+3} X_{j}^{(9)}\right| \geq\left|N[b] \biguplus N\left[x_{i+2}\right]\right| \geq 8$. Taking into account $\left|X_{i}^{(9)}\right| \geq 1$, we obtain $\left|\cup_{j=i}^{i+3} X_{j}^{(9)}\right| \geq 1+8=9$ contradicting the condition of the lemma. Thus, Lemma 4 is settled.

Lemma 5. If $S=\cup_{j=i}^{i+3} X_{j}^{(9)}$ (respectively, $S=\cup_{j=i}^{i+3} Y_{j}^{(9)}$ ) and $S^{\prime}=\cup_{j=i+4}^{i+7} X_{j}^{(9)}$ (respectively, $S^{\prime}=\cup_{j=i+4}^{i+7} Y_{j}^{(9)}$ ) are both scant, then $|S|=\left|S^{\prime}\right|=8$.

Proof. We show that the result holds for $S=\cup_{j=i}^{i+3} X_{j}^{(9)}, S^{\prime}=$ $\cup_{j=i+4}^{i+7} X_{j}^{(9)}$ as it follows analogously on $S=\cup_{j=i}^{i+3} Y_{j}^{(9)}$, $S^{\prime}=\cup_{j=i+4}^{i+7} Y_{j}^{(9)}$. By Lemma $4,\left(\cup_{j=i+1}^{i+3} X_{j}^{[6]}\right) \cup\left(\cup_{j=i+5}^{i+7} X_{j}^{[6]}\right)=$ $\varnothing$. 
Claim 1. $X_{i+4}^{[6]}=\varnothing$.

Proof of Claim 1. Suppose to the contrary that there exists $a \in X_{i+4}^{[6]}$. Remember that $Q_{\leq d}[a]$ is the set of all vertices in $G$ whose distance from $a \in V(G)$ is at most $d$ in $G$. Since $\delta(G) \geq \kappa(G)=3$ and $G$ is $C_{4}$-free, we have $\left|Q_{\leq 2}[a]\right| \geq 8$. Note that $Q_{\leq 2}[a] \subseteq \cup_{j=i+2}^{i+6} X_{j}^{[4,8]}$. Hence, $\left|\cup_{j=i+2}^{i+3} X_{j}^{[4,8]}\right|+$ $\left|\cup_{j=i+4}^{i+6} X_{j}^{[4,8]}\right| \geq Q_{\leq 2}[a] \geq 8$. There are two cases to consider:

Case 1: suppose that $\left|\cup_{j=i+4}^{i+6} X_{j}^{[4,8]}\right| \geq 3$. Note that $\left(\cup_{j=i+4}^{i+6} X_{j}^{[4,8]}\right) \cap\left(\cup_{j=i+4}^{i+7} X_{j}^{(2)}\right) \stackrel{j=i+4}{=} \varnothing$. Thus, upon making use of Lemma 2, we arrive at $\left|\cup_{j=i+4}^{i+7} X_{j}^{(9)}\right| \geq\left|\left(\cup_{j=i+4}^{i+6} X_{j}^{[4,8]}\right) \biguplus\left(\cup_{j=i+4}^{i+7} X_{j}^{(2)}\right)\right| \geq 3+6=$

9 immediately contradicting the condition that $S^{\prime}$ is scant.

Case 2: now, suppose that $\left|\cup_{j=i+4}^{i+6} X_{j}^{[4,8]}\right|<3$. Then, $\left|\cup_{j=i+2}^{i+3} X_{j}^{[4,8]}\right| \geq 6$, and since $\left(\cup_{j=i+2}^{i+3} X_{j}^{[4,8]}\right) \cap\left(\cup_{j=i}^{i+3}\right.$ $\left.X_{j}^{(2)}\right)=\varnothing$, we obtain, as a consequence of Lemma 2 , $\left|\cup_{j=i}^{i+3} X_{j}^{(9)}\right| \geq\left|\left(\cup_{j=i+2}^{i+3} X_{j}^{[4,8]}\right) \biguplus\left(\cup_{j=i}^{i+3} X_{j}^{(2)}\right)\right| \geq 6+6=12$

which contradicts the condition that $S$ is scant. Thus, $X_{i+4}^{(6)}=\varnothing$ and our claim is settled.

By Lemma 3, $S$ and $S^{\prime}$ are both detachable. In other words, there exist $u, v \in V(G)$ such that $H=S-u$ and $H^{\prime}=$ $S^{\prime}-v$ are both detached.

Claim 2. $u=x_{i+3}$ and $v=x_{i+4}$

Proof of Claim 2. Suppose to the contrary that $u \neq x_{i+3}$ or $v \neq x_{i+4}$. Observe that since $H=S-u\left(H^{\prime}=S^{\prime}-v\right)$ is a detached subgraph of $S\left(S^{\prime}\right)$, every path connecting some vertex in $X_{i}^{(9)}\left(X_{i+4}^{(9)}\right)$ to some vertex in $X_{i+3}^{(9)}\left(X_{i+7}^{(9)}\right)$ contains $u$ $(v)$. This means that every path connecting some vertex in $X_{i}^{(9)}$ to some vertex in $X_{i+7}^{(9)}$ contains $u$ and $v$. Thus, if $u \neq x_{i+3}$ $\left(v \neq x_{i+4}\right)$, since $H$ and $H^{\prime}$ are detached subgraphs of $S$ and $S^{\prime}$, respectively, and also noting that $\cup_{j=i+1}^{i+7} X_{j}^{[6]}=\varnothing$, we see that there is no path connecting $x_{i+3}\left(x_{i+4}\right)$ to $z$ in $G-\{u, v\}$ contradicting $\kappa(G)=3$. Hence, $u=x_{i+3}$ and $v=x_{i+4}$, settling our claim.

We now consider $|S|=\left|\cup_{j=i}^{i+3} X_{j}^{(9)}\right|$. If $\left|X_{j}^{(9)}\right|=1$ for $j \in\{i, i+1, i+2\}$, then $H=S-x_{j}$ is detached, contradicting Claim 2. Thus, $\left|X_{j}^{(9)}\right| \geq 2$ for $j \in\{i, i+1, i+2\}$. If $\left|X_{i+3}^{(9)}\right| \geq 3$, we obtain $\left|\cup_{j=i}^{i+3} X_{j}^{(9)}\right| \geq 2+2+2+3=9$ contradicting the condition of the lemma. Thus, $\left|X_{i+3}^{(9)}\right| \leq 2$. If $\left|X_{i+3}^{(9)}\right|=2$, we are done since $\left|\cup_{j=i}^{i+3} X_{j}^{(9)}\right| \geq 2+2+2+2=8$, so suppose $\left|X_{i+3}^{(9)}\right|=1$. This means that $\left|\cup_{j=i}^{i+3} X_{j}^{(9)}\right| \geq 7$, and thus, it remains to prove the following claim.

Claim 3. $\left|\cup_{j=i}^{i+3} X_{j}^{(9)}\right| \neq 7$

Proof of Claim 3. Suppose to the contrary that $\left|X_{j=i}^{(i+3)}\right|=7$. This means that $\left|X_{i}^{(9)}\right|=\left|X_{i+1}^{(9)}\right|=\left|X_{i+2}^{(9)}\right|=2$ and $\left|X_{i+3}^{(9)}\right|=1$. This yields $\left|\cup_{j=i}^{i+2} X_{j}^{(9)}\right|=6$ and $\left|\cup_{i=i+1}^{i+3} X_{j}^{(9)}\right|=5$. Let $a_{j}$, $j \in\{i, i+1, i+2\}$ be the vertex in $X_{j}^{(\overline{9})^{+}+1}$ which is not $x_{j}$. If $a_{j} \in X_{j}^{[9]}$, then $H=S-x_{j}$ is detached contradicting Claim 2. Thus, $a_{j} \in X_{j}^{(8)}$. Now if $a_{i+1} x_{i+1} \notin E(G)$, then since $a_{i+1}$ and $x_{i+1}$ can share at most one neighbour, we have $\left|\cup_{j=i}^{i+2} X_{j}^{(9)}\right| \geq\left|N\left[a_{i+1}\right] \cup N\left[x_{i+1}\right]\right| \geq 7$,

contradicting $\left|\cup_{j=i}^{i+2} X_{j}^{(9)}\right|=6$. A similar argument leads us to conclude that $a_{i+2} x_{i+2} \in E(G)$. Observe that $a_{i+2}$ is necessarily adjacent to at least a vertex in $X_{i+1}^{(9)}$. Note that if $a_{i+1} a_{i+2} \in E(G)$, then $\left\{a_{i+1} a_{i+2}, a_{i+2} x_{i+2}, x_{i+2} x_{i+1}, x_{i+1} a_{i+1}\right\}$ forms a $C_{4}$-subgraph which is illegal. Thus, $a_{i+2}$ can only be adjacent to $x_{i+1} \in X_{i+1}^{(9)}$ leading us to conclude that $a_{i+2} x_{i+1} \in E(G)$. Now, consider $x_{i+2}$. If $x_{i+2} a_{i+1} \in E(G)$, then $\left\{x_{i+2} a_{i+1}, a_{i+1} x_{i+1}, x_{i+1} a_{i+2}\right.$, $\left.a_{i+2} x_{i+2}\right\}$ would form $C_{4}$-subgraph which is illegal. Thus, $x_{i+2} a_{i+1} \notin E(G)$. Hence, $H=S-x_{i+1}$ is detached, contradicting Claim 2. Hence, Claim 3 is settled.

Thus, since $\left|\cup_{j=i}^{i+3} X_{j}^{(9)}\right| \geq 7$ and $\left|\cup_{j=i}^{i+3} X_{j}^{(9)}\right| \neq 7$, we have $\left|\cup_{j=i}^{i+3} X_{j}^{(9)}\right| \geq 8$. Therefore, since $S$ is scant, we conclude $\left|\cup_{j=i}^{i+3} X_{j}^{(9)}\right|=8$. A similar set of arguments leads us to conclude that $\left|S^{\prime}\right|=\left|\cup_{j=i+4}^{i+7} X_{j}^{(9)}\right|=8$, thereby settling Lemma 5.

\section{Lemma 6"}

(a) If $S=\cup_{j=i}^{i+3} X_{j}^{(9)}$ and $S^{\prime}=\cup_{j=i+4}^{i+7} X_{j}^{(9)}$ are both scant, then $S^{\prime \prime} \stackrel{j=i}{=} \cup_{j=i+8}^{i+11} X_{j}^{(9)}$ is not scant.

(b) If $S=\cup_{j=i}^{i+3} Y_{j}^{(9)}$ and $S^{\prime}=\cup_{j=i+4}^{i+7} Y_{j}^{(9)}$ are both scant, then $S^{\prime \prime}=\cup_{j=i+8}^{j+11} Y_{j}^{(9)}$ is not scant.

Proof. We show that the result holds for (a) as it follows analogously for (b). Suppose to the contrary that $S, S^{\prime}$, and $S^{\prime \prime}$ are all scant. By Lemma 4, $\left(\cup_{j=i+1}^{i+3} X_{j}^{[6]}\right) \cup\left(\cup_{j=i+5}^{i+7} X_{j}^{[6]}\right) \cup\left(\cup_{j=i+9}^{i+11} X_{j}^{[6]}\right)=\varnothing$. By Claim $1, X_{i+4}^{[6]} \cup X_{i+8}^{[6]}=\varnothing$; therefore, $\cup_{j=i+1}^{i+11} X_{j}^{[6]}=\varnothing$. Since $S, S^{\prime}$, and $S^{\prime \prime}$ are all scant, we know, by Lemma 3, that $S, S^{\prime}$, and $S^{\prime \prime}$ are all detachable. Observe that, by Claim 2, $H=S-x_{i+3}$ and $H^{\prime \prime}=S^{\prime \prime}-x_{i+8}$ are both detached. Thus, since $\cup_{j=i+1}^{i+11} X_{j}^{[6]}=\varnothing$, we have it that there is no path connecting $\left\{x_{i+4}, x_{i+5}, x_{i+6}, x_{i+7}\right\}$ to $z$ in $G-\left\{x_{i+3}, x_{i+8}\right\}$. Thus, $G-\left\{x_{i+3}, x_{i+8}\right\}$ is disconnected, contradicting $\kappa(G)=3$. This settles Lemma 6.

Throughout the remainder of this paper, we assume that $r-3 \equiv c \bmod 4, \quad c \in\{0,1,2,3\}$, and we define the set $I=\{10,11, \ldots, r-40-c / 4\}$.

Lemma 7. Let $l, p, q \in I$ such that $p+1<q$.

(a) If $S_{p}=\cup_{j=4 p}^{4 p+3} X_{j}^{(9)}$ and $S_{q}=\cup_{j=4 q}^{4 q+3} X_{j}^{(9)}$ are both scant and $S_{l}=\cup_{j=4 l}^{4 l+3} X_{j}^{(9)}$ is not scant whenever $p<l<q$, then there exists $d$ (where $p+1 \leq d \leq q-1$ ) such that $\left|\cup_{j=4 d}^{4 d+3} X_{j}^{(9)}\right| \geq 12$ or $\left|\cup_{j=4 d-4}^{4 d+3} X_{j}^{(9)}\right| \geq 21$ or $\mid \cup_{j=4 d}^{4 d+7}$ $X_{j}^{(9)} \mid \geq 21$.

(b) If $S_{p}=\cup_{j=4 p}^{4 p+3} Y_{j}^{(9)}$ and $S_{q}=\cup_{j=4 q}^{4 q+3} Y_{j}^{(9)}$ are both scant and $S_{l}=\cup_{j=4 l}^{4 l+3} Y_{j}^{(9)}$ is not scant whenever $p<l<q$, 
then there exists $d$ (where $p+1 \leq d \leq q-1$ ) such that $\left|\cup_{j=4 d}^{4 d+3} Y_{j}^{(9)}\right| \geq 12$ or $\left|\cup_{j=4 d-4}^{4 d+3} Y_{j}^{(9)}\right| \geq 21$ or $\left|\cup_{j=4 d}^{4 d+7} Y_{j}^{(9)}\right| \geq$ 21.

Proof. We show that the result holds for (a) as it follows analogously for (b). From Lemma 4, we have $\left(\cup_{j=4 p+1}^{4 p+3} X_{j}^{[6]}\right) \cup\left(\cup_{j=4 q+1}^{4 q+3} X_{j}^{[6]}\right)=\varnothing$. Suppose there exists a vertex $a \in X_{4 q}^{[6]}$. Remember that $\left|Q_{\leq 2}[a]\right| \geq 8$. Consider $\left|Q_{\leq 3}[a]\right|$. If $\left|Q_{\leq 3}[a]\right| \leq 8$, then $Q_{\leq 3}[a]-Q_{\leq 2}[a]=\varnothing$ and there would be no path from $a$ to $z$ contradicting the condition that $G$ is connected. Thus, $\left|Q_{\leq 3}[a]\right| \geq 9$. Since $a \in X_{4 q}^{[6]}$, we have $Q_{\leq 3}[a] \subseteq \cup_{j=4 q-3}^{4 q+3} X_{j}^{[3,9]}$ and consequently $\left|\cup_{j=4 q-3}^{4 q+3} X_{j}^{[3,9]}\right| \geq\left|Q_{\leq 3}[a]\right| \geq 9$. Note that $\left(\cup_{j=4 q}^{4 q+3} X_{j}^{[3,9]}\right) \cap$ $\left(\cup_{j=4 q}^{4 q+3} X_{j}^{(2)}\right)=\varnothing$; furthermore, $S_{q}=\cup_{j=4 q}^{4 q+3} X_{j}^{(9)}$ is scant. Thus, making use of Lemma 2 , we obtain $\left|\cup_{j=4 q}^{4 q+3} X_{j}^{[3,9]}\right| \leq 2$. This gives us $\left|\cup_{j=4 q-3}^{4 q-1} X_{j}^{[3,9]}\right| \geq 7$, and using Lemma 2 again, we obtain $\left|\cup_{j=4 q-3}^{4 q-1} X_{j}^{(9)}\right| \geq\left|\left(\cup_{j=4 q-4}^{4 q-1} X_{j}^{[3,9]}\right) \biguplus\left(\cup_{j=4 q-4}^{4 q-1} X_{j}^{(2)}\right)\right| \geq$ $7+6=13$ and we are done. So, for the rest of this lemma, we will assume $X_{4 q}^{[6]}=\varnothing$.

Claim 4. There exists a vertex $a \in \cup_{j=4 p+4}^{4 q-1} X_{j}^{[6]}$.

Proof of Claim 4. Suppose to the contrary that $\cup_{j=4 p+4}^{4 q-1} X_{j}^{[6]}=\varnothing$, then $\cup_{j=4 p+1}^{4 q+3} X_{j}^{[6]}=\varnothing$. Since $S_{p}$ and $S_{q}$ are both scant, we have it, by Lemma 2 , that $S_{p}$ and $S_{q}$ are both detachable. Thus, there exist $u$ and $v$ such that $H_{p}=S_{p}-u$ and $H_{q}=S_{q}-v$ are both detached. Thus, there is no path in $G-\{u, v\}$ connecting $\left\{x_{4 p+4}, x_{4 p+5}, \ldots, x_{4 q-1}\right\}$ to $z$. Thus, $G-\{u, v\}$ is disconnected, contradicting $\kappa(G)=3$ and therefore Claim 4 is settled.

Now we have it, by Claim 4, that there exists $d \in\{p+1, p+2, \ldots, q-1\}$ such that $a \in \cup_{j=4 d}^{4 d+3} X_{j}^{[6]}$. With this in mind, there are four cases to consider.

Case 1: suppose $a \in X_{4 d}^{[6]}$. Since $Q_{\leq 3}[a] \subseteq \cup_{j=4 d-3}^{4 d+3} X_{j}^{[3,9]}$ and $\left(\cup_{j=4 d-3}^{4 d+3} X_{j}^{[3,9]}\right) \cap\left(\cup_{j=4 d-4}^{4 d+3} X_{j}^{(2)}\right)=\varnothing$, we have, by Lemma 2 and $\left|Q_{\leq 3}[a]\right| \geq 9$, that

$$
\begin{aligned}
\left|\cup_{j=4 d-4}^{4 d+3} X_{j}^{(9)}\right| & \geq\left|\cup_{j=4 d-3}^{4 d+3} X_{j}^{[3,9]}\right|+\left|\cup_{j=4 d-4}^{4 d+3} X_{j}^{(2)}\right| \geq\left|Q_{\leq 3}[a]\right| \\
& +\left|\cup_{j=4 d-4}^{4 d-1} X_{j}^{(2)}\right|+\left|\cup_{j=4 d}^{4 d+3} X_{j}^{(2)}\right| \geq 9+6+6=21 .
\end{aligned}
$$

Case 2: $a \in X_{4 d+1}^{[6]}$. It is obvious that $T(z, a) \cap N_{4 d-2}$ is nonempty; hence, there exists a vertex $b \in T(z, a) \cap$ $N_{4 d-2}$. Observe that $d_{G}(a, b)=d_{G}(z, a)-d_{G}(z, b)=$ $(4 d+1)-(4 d-2)=3$. Thus, since $a \in X_{4 d+1}^{[6]}$, we have $b \in X_{4 d-2}^{[3,9]}$ and thus $\left|X_{4 d-2}^{[3,9]}\right| \geq 1$. Furthermore, $Q_{\leq 2}[a] \subseteq \cup_{j=4 d-1}^{4 d+3} X_{j}^{[4,8]} \quad$ and $\quad\left(\cup_{j=4 d-1}^{4 d+3} X_{j}^{[4,8]}\right) \cap$ $\left(\cup_{j=4 d-4}^{4 d+3} X_{j}^{(2)}\right)=\varnothing$. Hence, by Lemma 2,

$$
\begin{aligned}
\left|\cup_{j=4 d-4}^{4 d+3} X_{j}^{(9)}\right| & \geq\left|X_{4 d-2}^{[3,9]}\right|+\left|\cup_{j=4 d-1}^{4 d+3} X_{j}^{[4,8]}\right|+\left|\cup_{j=4 d-4}^{4 d-1} X_{j}^{(2)}\right| \\
& +\left|\cup_{j=4 d}^{4 d+3} X_{j}^{(2)}\right| \geq 1+8+6+6=21 .
\end{aligned}
$$

Case 3: $a \in X_{4 d+2}^{[6]}$. Note that $a$ is necessarily adjacent to at least one vertex, say $b$, in $X_{4 d+1}^{(9)}$. More specifically, $b \in X_{4 d+1}^{[5,7]}$. Observe that $N(a) \cup N(b) \subseteq$ $\cup_{j=4 d}^{4 d+3} X_{j}^{[4,8]}$ and $\left(\cup_{j=4 d}^{4 d+3} X_{j}^{[4,8]}\right) \cap\left(\cup_{j=4 d}^{4 d+3} X_{j}^{(2)}\right)=\varnothing$. Thus, if $a$ and $b$ do not share any neighbours, we are done, by Lemma 2 , since $\left|\cup_{j=4 d}^{4 d+3} X_{j}^{(9)}\right| \geq\left|\cup_{j=4 d}^{4 d+3} X_{j}^{[4,8]}\right|+$ $\left|\cup_{j=4 d}^{4 d+3} X_{j}^{(2)}\right| \geq|N(a) \cup N(b)|+\left|\cup_{j=4 d}^{4 d+3} X_{j}^{(2)}\right| \geq 6+6=12$. So, suppose $a$ and $b$ share a neighbour, say $c$. Note that $c \notin X_{4 d}^{(9)} \cup X_{4 d+3}^{(9)}$ since $\left(X_{4 d}^{(9)} \cap N(a)\right) \cup\left(X_{4 d+3}^{(9)} \cap\right.$ $N(b))=\varnothing$. Thus, $\{a, b, c\} \subseteq X_{4 d+1}^{[4,8]} \cup X_{4 d+2}^{[4,8]}$. Since $G$ is $C_{4}$-free, any pair of vertices can only share at most one neighbour. Recognise that $a$ and $b$ share $c$, also $b$ and $c$ share $a$, and finally $a$ and $c$ share $b$. Furthermore, $\operatorname{deg}(a), \operatorname{deg}(b), \operatorname{deg}(c) \geq 3$; thus, $a$ is adjacent to another vertex, say $a^{\prime}, b$ is adjacent to another vertex, say $b^{\prime}$, and $c$ is adjacent to another vertex, say $c^{\prime}$, such that $a^{\prime} \neq b^{\prime} \neq c^{\prime} \neq a^{\prime}$. Thus, since $\left\{a, a^{\prime}, b, b^{\prime}, c, c^{\prime}\right\} \subseteq N$ $(a) \cup N(b) \cup N(c) \subseteq \cup_{j=4 d}^{4 d+3} X_{j}^{[3,9]}$ and $\left(\cup_{j=4 d}^{4 d+3} X_{j}^{[3,9]}\right) \cap$ $\left(\cup_{j=4 d}^{4 d+3} X_{j}^{(2)}\right)=\varnothing$, we obtain, by Lemma 2, $\left|\cup_{j=4 d}^{4 d+3} X_{j}^{(9)}\right| \geq\left|\cup_{j=4 d}^{4 d+3} X_{j}^{(2)}\right|+\left|\cup_{j=4 d}^{4 d+3} X_{j}^{[3,9]}\right| \geq 6+6=12$.

(i) Case 4: $a \in X_{4 d+3}^{[6]}$. Since $Q_{\leq 3}[a] \subseteq \cup_{j=4 d}^{4 d+6} X_{j}^{[3,9]}$ and $\left(\cup_{j=4 d}^{4 d+6} X_{j}^{[3,9]}\right) \cap\left(\cup_{j=4 d}^{4 d+7} X_{j}^{(2)}\right)$, we have, by Lemma 2 and $\left|Q_{\leq 3}[a]\right| \geq 9$, that

$$
\begin{aligned}
\left|\cup_{j=4 d}^{4 d+7} X_{j}^{(9)}\right| & \geq\left|\cup_{j=4 d}^{4 d+7} X_{j}^{[3,9]}\right|+\left|\cup_{j=4 d}^{4 d+7} X_{j}^{(2)}\right| \geq\left|Q_{\leq 3}[a]\right| \\
& +\left|\cup_{j=4 d}^{4 d+3} X_{j}^{(2)}\right|+\left|\cup_{j=4 d+4}^{4 d++7} X_{j}^{(2)}\right| \geq 9+6+6=21 .
\end{aligned}
$$

Thus, Lemma 7 is settled.

Throughout the remainder of this paper, for $p \in I$, we define $S_{p}=\cup_{j=4 p}^{4 p+3} X_{j}^{(9)}$ (respectively, $S_{p}^{\prime}=\cup_{j=4 p}^{4 p+3} Y_{j}^{(9)}$ ) and $s_{p}=\left|S_{p}\right|$ (respectively, $s_{p}^{\prime}=\left|S_{p}^{\prime}\right|$ ).

Lemma 8. Let $p, q \in I$.

(a) If $S_{p}$ and $S_{q}$ are both scant and $S_{l}$ is not scant whenever $l \in\{p+2, p+3, \ldots, q-1\}$, then $\sum_{j=p}^{q-1} s_{p} \geq 9(q-p)$.

(b) If $S_{p}^{\prime}$ and $S_{q}^{\prime}$ are both scant and $S_{l}^{\prime}$ is not scant whenever $l \in\{p+2, p+3, \ldots, q-1\}$, then $\sum_{j=p}^{q-1} s_{p}^{\prime} \geq 9(q-p)$.

Proof. We show that the result holds for (a) as it follows analogously for (b). By Lemma 7, there exists $d \in\{p+1, p+$ 
$2, \ldots, q-1\}$ such that $s_{d} \geq 12$ or $s_{d-1}+s_{d} \geq 21$ or $s_{d}+s_{d+1} \geq 21$. Note that $s_{p}+s_{p+1} \geq 15$ since if $S_{p+1}$ is scant, we obtain, by Lemma $5, s_{p}+s_{p+1}=8+8=16>15$, otherwise we have, by Lemma $2, s_{p}+s_{p+1} \geq 6+9=15$.

(i) Case $1: d \in\{p+3, p+4, \ldots, q-2\}$. Since $s_{p}+s_{p+1} \geq$ 15 and $s_{l} \geq 9$ whenever $l \in\{p+2, p+3, \ldots, q-1\}$, we have the following. If $s_{d} \geq 12$, then

$$
\begin{aligned}
\sum_{j=p}^{q-1} s_{j} & \geq\left(s_{p}+s_{p+1}\right)+\left(\sum_{j=p+2}^{d-1} s_{j}\right)+s_{d}+\left(\sum_{j=d+1}^{q-1} s_{j}\right) \geq 15 \\
& +9(d-1-(p+1))+12+9(q-1-d)=9(q-p) .
\end{aligned}
$$

If $s_{d-1}+s_{d} \geq 21$, then

$$
\begin{aligned}
\sum_{j=p}^{q-1} s_{j} & \geq\left(s_{p}+s_{p+1}\right)+\left(\sum_{j=p+2}^{d-2} s_{j}\right)+\left(s_{d-1} s_{d}\right)+\left(\sum_{j=d+1}^{q-1} s_{j}\right) \\
& \geq 15+9(d-2-(p+1))+21+9(q-1-d)=9(q-p) .
\end{aligned}
$$

(i) If $s_{d}+s_{d+1} \geq 21$, then

$$
\begin{aligned}
\sum_{j=p}^{q-1} s_{j} & \geq\left(s_{p}+s_{p+1}\right)+\left(\sum_{j=p+2}^{d-1} s_{j}\right)+\left(s_{d}+s_{d+1}\right)+\left(\sum_{j=d+2}^{q-1} s_{j}\right) \\
& \geq 15+9(d-1-(p+1))+21+9(q-1 \\
& -(d+1))=9(q-p) .
\end{aligned}
$$

Case 2: $d=p+1$. It is obvious in this case that $S_{p+1}$ is not scant, thus making use of the condition of the current lemma, and $s_{l} \geq 9$ whenever $l \in\{p+1, p+3, \ldots, q-1\}$. Note that, by Lemma 2 , $s_{p} \geq 6$. If $s_{d} \geq 12$, then

$$
\begin{aligned}
\sum_{j=p}^{q-1} s_{j} & =s_{p}+s_{p+1(=d)}+\sum_{j=p+2}^{q-1} s_{j} \geq 6+12+9(q-1-(p+1)) \\
& =9(q-p) .
\end{aligned}
$$

$$
\begin{aligned}
& \text { If } s_{d-1}+s_{d} \geq 21, \text { then } \\
& \begin{aligned}
\sum_{j=p}^{q-1} s_{j} & =\left(s_{p(=d-1)}+s_{p+1(=d)}\right)+\sum_{j=p+2}^{q-1} s_{j} \geq 21+9(q-1-(p+1)) \\
& =9(q-p)+3 .
\end{aligned}
\end{aligned}
$$

$$
\begin{aligned}
& \text { If } s_{d}+s_{d+1} \geq 21 \text {, then } \\
& \begin{array}{l}
\sum_{j=p}^{q-1} s_{j}=s_{p}+s_{p+1(=d)}+s_{p+2(=d+1)}+\sum_{j=p+3}^{q-1} s_{j} \geq 6+21 \\
\quad+9(q-1-(p+2))=9(q-p) .
\end{array}
\end{aligned}
$$

Case 3: $d=p+2$. If $s_{d} \geq 12$, then

$$
\begin{aligned}
\sum_{j=p}^{q-1} s_{j}= & \left(s_{p}+s_{p+1}\right)+s_{p+2(=d)}+\sum_{j=p+3}^{q-1} s_{j} \geq 15+12 \\
& +9(q-1-(p+2))=9(q-p) .
\end{aligned}
$$

If $s_{d-1}+s_{d} \geq 21$, then

$$
\begin{aligned}
\sum_{j=p}^{q-1} s_{j}= & s_{p}+\left(s_{p+1(=d-1)}+s_{p+2(=d)}\right)+\sum_{j=p+3}^{q-1} s_{j} \geq 6+21 \\
& +9(q-1-(p+2))=9(q-p) .
\end{aligned}
$$

If $s_{d}+s_{d+1} \geq 12$, then

$$
\begin{aligned}
\sum_{j=p}^{q-1} s_{j}= & \left(s_{p}+s_{p+1}\right)+s_{p+2(=d)}+s_{p+3(=d+1)}+\sum_{j=p+4}^{q-1} s_{j} \geq 15 \\
& +21+9(q-1-(p+3))=9(q-p) .
\end{aligned}
$$

Case 4: $d=q-1$. If $s_{d} \geq 12$, then

$$
\begin{aligned}
\sum_{j=p}^{q-1} s_{j}= & \left(s_{p}+s_{p+1(=d)}\right)+\left(\sum_{j=p+2}^{q-2} s_{j}\right)+s_{q-1} \geq 15 \\
& +9(q-2-(p+1))+12=9(q-p) .
\end{aligned}
$$

If $s_{d-1}+s_{d} \geq 21$, then

$$
\begin{aligned}
\sum_{j=p}^{q-1} s_{j}= & \left(s_{p}+s_{p+1}\right)+\left(\sum_{j=p+2}^{q-3} s_{j}\right)+\left(s_{(q-2=d-1)}+s_{(q-1=d)} \geq 15\right. \\
& +9(q-3-(p+1)))+21=9(q-p) .
\end{aligned}
$$

If $s_{d}+s_{d+1} \geq 21$, we have the following. Since $S_{q}$ is scant, we have $s_{q} \leq 8$ and, therefore, $s_{q-1} \geq 13$. Then,

$$
\begin{aligned}
\sum_{j=p}^{q-1} s_{j}= & \left(s_{p}+s_{p+1}\right)+\left(\sum_{j=p+2}^{q-2} s_{j}\right)+s_{q-1} \geq 15 \\
& +9(q-2-(p+1))+13=9(q-p)+1 .
\end{aligned}
$$

Thus, Lemma 8 is settled.

\section{Lemma 9"}

(a) $\sum_{j \in I} s_{j} \geq 9|I|-3$.

(b) $\sum_{j \in I} s_{j}^{\prime} \geq 9|I|-3$.

Proof. We show that the result holds for (a) as it follows analogously for (b). If $S_{j}$ is not scant for all $j \in I$, then $s_{j} \geq 9$ for all $j \in I$ and $\sum_{j \in I} s_{j} \geq 9|I|$. If there exists only one $p \in I$ such that $S_{p}$ is scant, then $\sum_{j \in I} s_{j}=\sum_{j \in I-\{p\}} s_{j}+$ $s_{p} \geq 9(|I|-1)+6=9|I|-3$. If there exist at least two $p \in|I|$ 
such that $S_{p}$ is scant, then we have the following. Let $p_{1}, p_{2}, \ldots, p_{m} \in \Theta_{1}$ such that $S_{p_{j}}$ is scant whenever $j \in\{1,2, \ldots, m\}$. Define $\Theta_{2}=\left\{p_{j} \in \Theta_{1}: p_{j-1}=p_{j}-1\right\}$ and $\Theta_{3}=\Theta_{1}-\Theta_{2}$. We denote the elements of $\Theta_{3}$ as $q_{1}, q_{2}, \ldots, q_{f}$, where $q_{1}<q_{2}<\cdots<q_{f}$. Let $I^{\prime}=\left\{q_{1}, q_{1}+1, q_{1}+2, \ldots\right.$, $\left.q_{f}-1, q_{f}\right\}$. Thus, applying Lemma 2 and Lemma 8 , we obtain

$$
\begin{aligned}
\sum_{j \in I} s_{j}= & \left(\sum_{j \in I-I^{\prime}} s_{j}\right)+\left(\sum_{j \in I^{\prime}-\left\{q_{f}\right\}} s_{j}\right)+s_{q_{f}} \geq 9\left(|I|-\left|I^{\prime}\right|\right) \\
& +\sum_{i=1}^{f-1}\left(\sum_{j=q_{i}}^{q_{i+1}-1} s_{j}\right)+6 \geq 9\left(|I|-\left(q_{f}-\left(q_{1}-1\right)\right)\right) \\
& +\sum_{i=1}^{f-1} 9\left(q_{i+1}-q_{i}\right)+6 \geq 9|I|-9\left(q_{f}-q_{1}\right)-3 \\
& +9\left(q_{f}-q_{1}\right)=9|I|-3 .
\end{aligned}
$$

Theorem 2. Let $G$ be a 3-vertex-connected $C_{4}$-free graph of order $n$. Then, $\operatorname{rad}(G) \leq(2 n / 9)+(263 / 9)$.

Proof. Remember that $k_{i}=\left|N_{i}\right|, 0 \leq i \leq r$. Note that, since $G$ is 3 -vertex-connected, $k_{i} \geq 3,0<i<r$. Note also that, since $N_{0}, N_{r} \neq \varnothing, k_{0}, k_{r} \geq 1,0<i<r$. Thus, by 1 and Lemma 9, we obtain

$$
\begin{aligned}
n= & \left|\cup_{j=0}^{r} N_{j}\right|=1+\left|\cup_{j=1}^{39} N_{j}\right|+\left|\cup_{j=40}^{r-37-c} N_{j}\right|+\left|\cup_{r-36-c}^{r} N_{j}\right| \geq\left|\cup_{j=0}^{39} N_{j}\right| \\
& +\left|\cup_{j=40}^{r-37-c} X_{j}^{(9)}\right|+\left|\cup_{j=40}^{r-37-c} Y_{j}^{(9)}\right|+\left|\cup_{r-36-c}^{r-1} N_{j}\right|+1 \geq 2 \\
& +\sum_{j=1}^{39} k_{j}+\sum_{j \in I} s_{j}+\sum_{j \in I} s_{j}^{\prime} \\
& +\sum_{r-36-c}^{r} k_{j} \geq 3 \cdot 40+(9|I|-3)+(9|I|-3)+3 \cdot(36+c) \\
= & 18|I|+221+c=18\left(\frac{r-40-c}{4}-9\right)+221+c \\
= & \frac{9 r}{2}-121-\frac{7 c}{2} \geq \frac{9 r}{2}-121-\frac{21}{2}=\frac{9 r}{2}-\frac{263}{2} .
\end{aligned}
$$

Thus, $\operatorname{rad}(G) \leq(2 n / 9)+(263 / 9)$ as desired.

To show that this bound is asymptotically sharp, consider the graph described immediately following Theorem 1 .

\section{Data Availability}

No data were used to support this study.

\section{Disclosure}

The results in this paper are part of the first author's MPhilSc thesis.

\section{Conflicts of Interest}

The authors declare that they have no conflicts of interest.

\section{References}

[1] G. Chartrand and O. R. Oellermann, Applied and Algorithmic Graph Theory, McGraw-Hill, New York, NY, USA, 1993.

[2] P. Erdős, J. Pach, R. Pollack, and Z. Tuza, "Radius, diameter and minimum degree," Journal of Combinatorial Theory, Series B, vol. 47, no. 1, pp. 73-79, 1989.

[3] P. Dankelmann, G. Dlamini, and H. C. Swart, "Upper bounds on distance measures in $K_{2, l}$-free graphs," (In press), 2020.

[4] P. Dankelmann, S. Mukwembi, and H. C. Swart, "An upper bound on the radius of a 3-edge-connected graph," Utilitas Mathematica, vol. 73, 2007 (to appear).

[5] S. Mukwembi, "The radius triangle-free graphs with prescribed edge-connectivity," Utilitas Mathematica, vol. 77, pp. 135-144, 2008.

[6] J. Harant and H. Walther, "On the radius of Graphs," Journal of Combinatorial Theory, Series B, vol. 30, no. 1, pp. 113-117, 1981.

[7] J. Harant, "An upper bound for the radius of a 3-connected graph," Discrete Mathematics, vol. 122, no. 1-3, pp. 335-341, 1993. 